\title{
SPATIAL NOISE CANCELLATION INSIDE CARS: PERFORMANCE ANALYSIS AND EXPERIMENTAL RESULTS
}

\author{
Hanchi Chen, Prasanga Samarasinghe, Thushara D. Abhayapala, Wen Zhang \\ Research School of Engineering \\ College of Engineering and Computer Science \\ The Australian National University
}

\begin{abstract}
A loudspeaker array is a key component in active noise cancellation (ANC) systems. Most in-car ANC systems utilize the car's own integrated loudspeakers to cancel the noise due to engine and other sources. In this paper, we evaluate the integrated loudspeakers' noise cancelling capabilities by analyzing the in-car noise field and the loudspeaker responses. We show that the average noise power in a spatial region can be expressed using a series of coefficients, and that the noise field can be decomposed into several basis noise patterns. Through analysing the measurements in a car, we show that the car's built-in loudspeakers are capable of attenuating the driving noise by up to $30 \mathrm{~dB}$ for frequencies up to $500 \mathrm{~Hz}$ within a spherical region of $10 \mathrm{~cm}$ radius.
\end{abstract}

Index Terms - Active noise cancellation, spherical harmonics, loudspeaker array, sound field analysis

\section{INTRODUCTION}

The application of noise cancellation methods to minimize interior cabin noise has been a key topic of research in the automobile industry for the last $15-20$ years. Initially, this problem was approached via passive noise cancellation methods, which use acoustic treatments such as structural damping and acoustic absorption. However, with the growing need to improve fuel efficiency, there has been more preference on lighter bodies and smaller engines, which has significantly increased the structural vibration and consequent interior noise, predominantly at low frequencies (e.g. $0-500 \mathrm{~Hz}$ ) [1]. As passive methods were least effective with low frequency noise, active methods were developed where secondary loudspeakers were proposed to attenuate measured noise inside the cabin $[1,2,3,4,5]$. With modern in-car entertainment systems providing $4-6$ built-in loudspeakers, the addition of an active noise cancellation systems is considered to involve no greater cost [6].

Generally, active control creates a signal out of phase with that generated by the vehicle, thus noise is attenuated via destructive interference. In practice, this is achieved by using microphones to measure the residual difference between the two signals, and a feed forward/feed back control system to do the necessary processing [7]. Feed-forward systems use an additional "reference signal" correlated with the noise signal to attenuate them individually, whereas Feed-back systems use a single-input single-output system to attenuate overall measured noise [1]. Even though both methods are proven to deliver positive results, significant soundfield control over a single measurement point is only capable of narrowband attenuation at about $40 \mathrm{~Hz}$ [8]. Addressing this issue, multi-input multioutput (MIMO) controllers with multiple microphones as error sensors (typically mounted on headrests) were introduced to increase spatial coverage $[6,8,9,10,11,12]$. For the specific case of road noise cancellation, MIMO controllers were shown to achieve attenuation levels up to $8 \mathrm{~dB}$ below $40 \mathrm{~Hz}$ and around $3 \mathrm{~dB}$ within $80-200 \mathrm{~Hz}$ [8]. Recent work on non-automobile related MIMO controllers is presented in [13], where noise reduction above $10 \mathrm{~dB}$ is obtained over a distributed set of 16 spatial samples in $0.3 \times 0.3 \mathrm{~m}$ region for frequencies up to $500 \mathrm{~Hz}$. A wave domain MIMO ANC system is proposed in [14].

To the best of our knowledge, the existing in-car MIMO controllers are constrained to a set of arbitrary observation points. As a result, spatial control over continuous regions is limited and made worse with increased frequency. Addressing this issue, we focus this work on modeling vehicle-interior noise over a continuous spatial region such that noise control can be achieved over the region with size similar to a human head for frequencies up to $f=500 \mathrm{~Hz}$. We also derive the maximum attenuation levels for a given speaker arrangement so that industrial designers can investigate the potential noise cancellation capability of a given loudspeaker system for various noise sources and driving conditions. All of the analysis we perform are based on acoustic measurements taken in a real in-car environment.

The paper is organized as follows. Section 2 defines the problem and Section 3 describes the concept of average spatial noise. In Section 4 the performance analysis of a given speaker system is analyzed. Finally, Section 5 discusses experimental results based on real measurements.

\section{PROBLEM FORMULATION}

Denote the unwanted noise pressure at a point $\boldsymbol{x}$ as $P_{\mathrm{n}}(\boldsymbol{x})$, and the sound pressure due to the loudspeakers as $P_{\mathrm{c}}(\boldsymbol{x})$, the average residual noise energy within the interested region $S$ can be expressed as

$$
\int_{S}\left|P_{\mathrm{r}}(\boldsymbol{x})\right|^{2} d S=\int_{S}\left|P_{\mathrm{n}}(\boldsymbol{x})+P_{\mathrm{c}}(\boldsymbol{x})\right|^{2} d S .
$$

A complete in-car active noise cancellation system consists of many components, each component may have an impact on the system's overall performance. In this paper, we aim to evaluate the potential performance of in-car loudspeakers on ANC applications by estimating the minimum values of $\int_{S}\left|P_{\mathrm{r}}(\boldsymbol{x})\right|^{2} d S$ for various frequencies, based on the information on in-car noise field and the acoustic characteristics of the car's integrated loudspeakers, so as to see if the integrated loudspeakers are the potential bottleneck for in-car ANC systems. 


\section{PRELIMINARY: AVERAGE NOISE POWER OVER REGION}

We use the spherical harmonic decomposition to express the sound pressure at a given point within a region of interest. It is assumed that the region of interest $S$ with radius $R$ is a free space with no sound sources inside. The sound waves propagating inside the region are only due to sources outside the region. If we define a spherical coordinate system with its origin located at the center of $S$, the sound pressure $P(r, \vartheta, \varphi, k)$ at a given point and frequency within the sphere can be represented as a weighted sum of spherical harmonics $[15,16]$,

$$
P(r, \vartheta, \varphi, k)=\sum_{n=0}^{\infty} \sum_{m=-n}^{n} \alpha_{n m}(k) j_{n}(k r) Y_{n m}(\vartheta, \varphi),
$$

where $k=2 \pi f / c$ is the wave number, $f$ and $c$ are the frequency and the wave propagation speed, respectively. $\alpha_{n m}$ are the spherical harmonic coefficients, $j_{n}(k r)$ is the spherical Bessel function of order $n$, and $Y_{n m}(\vartheta, \varphi)$ denotes the spherical harmonic of order $n$ and degree $m . Y_{n m}(\vartheta, \varphi)$ has the orthogonal property

$$
\int_{0}^{\pi} \int_{0}^{2 \pi} Y_{n m} Y_{n^{\prime} m^{\prime}}^{*} \sin (\theta) d \vartheta d \varphi=\delta_{n-n^{\prime}, m-m^{\prime}}
$$

where the arguments $(\vartheta, \varphi)$ are omitted for simplicity, and $\delta_{n, m}$ is the two dimensional Dirac Delta function. We can then use the decomposition (2) to express the average sound energy within $S$. Due to the orthogonal property (3), we have

$$
\begin{aligned}
\int_{S}|P(\boldsymbol{x})|^{2} d S & =\int_{0}^{R} \int_{0}^{\pi} \int_{0}^{2 \pi} P(\boldsymbol{x}) P(\boldsymbol{x})^{*} r^{2} d r \sin (\theta) d \theta d \phi \\
& =\sum_{n, m} \alpha_{n m} \alpha_{n m}^{*} \int_{0}^{R} j_{n}^{2}(k r) r^{2} d r
\end{aligned}
$$

where $\boldsymbol{x}=(r, \theta, \phi)$, and the wave number $k$ is omitted for simplicity. Define a new symbol $W_{n}$, such that

$$
W_{n}=\left(\int_{0}^{R} j_{n}(k r)^{2} r^{2} d r\right)^{1 / 2} .
$$

Clearly $W_{n}$ is real, thus (5) becomes

$$
\int_{S}|P(\boldsymbol{x})|^{2} d S=\sum_{n, m}\left|\alpha_{n m} W_{n}\right|^{2},
$$

which shows that the average sound power level within $S$ is equal to the sum of squared spherical harmonic coefficients with weighing $W_{n}$.

In the active noise cancellation scenario, the residual noise field $P_{r}(\boldsymbol{x})$ in (1) thus have the average energy

$$
\begin{aligned}
\int_{S}\left|P_{\mathrm{r}}(\boldsymbol{x})\right|^{2} d S & =\int_{S}\left|P_{\mathrm{n}}(\boldsymbol{x})+P_{\mathrm{c}}(\boldsymbol{x})\right|^{2} d S \\
& =\sum_{n, m}\left|\left(\alpha_{n m}^{(\mathrm{n})}+\alpha_{n m}^{(\mathrm{c})}\right) W_{n}\right|^{2},
\end{aligned}
$$

where $\alpha_{n m}^{(\mathrm{n})}$ and $\alpha_{n m}^{(\mathrm{c})}$ are the spherical harmonic coefficients representing the noise field and the loudspeaker sound field, respectively.

We then move on to derive an estimation of $\int_{S}\left|P_{\mathrm{r}}(\boldsymbol{x})\right|^{2} d S$, by analyzing the noise field and loudspeaker channel characteristics.

\section{NOISE CANCELLING PERFORMANCE ANALYSIS}

\subsection{Noise field characterization}

For a certain driving condition, we assume that the random noise field within $S$ can be seen as a weighed combination of a number of fixed, basis noise patterns, or noise modes [17], each driving condition may have a different set of basis. Then the noise field pressure within $S$ at any time under a fixed driving condition can be decomposed as

$$
P_{\mathrm{n}}(\boldsymbol{x})=\sum_{i} g_{i} P_{i}(\boldsymbol{x})
$$

where $P_{i}(\boldsymbol{x})$ denotes the $i$ th basic noise pattern at $\boldsymbol{x}$, and $g_{i}$ are some random weighing factors for each noise pattern. Theoretically an infinite number of modes are needed to fully describe an arbitrary noise field, however for a relatively small region and low frequencies, only a small number of noise modes are required for a good approximation of the noise field [17]. Using the spherical harmonics decomposition (2) to decompose the noise field $P_{\mathrm{n}}(\boldsymbol{x})$ and the basis patterns $P_{i}(\boldsymbol{x})$, we can express each noise field coefficient $\alpha_{n m}^{(\mathrm{n})}$ using the corresponding coefficient $\alpha_{n m}^{i}$ of every basis pattern,

$$
\alpha_{n m}^{(\mathrm{n})}=\sum_{i} g_{i} \alpha_{n m}^{i} .
$$

We can write all the coefficients in a vector form such that $\boldsymbol{a}=$ $\left[\alpha_{00}^{(\mathrm{n})}, \alpha_{1-1}^{(\mathrm{n})}, . .\right]^{T}$, and $\boldsymbol{a}_{i}=\left[\alpha_{00}^{i}, \alpha_{1-1}^{i}, . .\right]^{T}$, then from (11) we have

$$
\boldsymbol{a}=\sum_{i} g_{i} \boldsymbol{a}_{i}
$$

In order to reflect the relative impact of each spherical harmonic coefficient on the overall noise level within $S$, we define the weighed coefficient vector $\boldsymbol{c}_{i}=\left[\alpha_{00}^{i} W_{0}, \alpha_{1-1}^{i} W_{1}, . .\right]^{T}$, and by multiplying both sides of (12) with a diagonal matrix $\boldsymbol{W}$ with $\operatorname{diag}\{\boldsymbol{W}\}=$ $\left[W_{0}, W_{1}, W_{1}, W_{1}, W_{2}, \ldots\right]^{T}$, we have

$$
\boldsymbol{c}=\sum_{i} g_{i} \boldsymbol{c}_{i}
$$

where $\boldsymbol{c}$ represents the random noise field in $S$ and $\|\boldsymbol{c}\|^{2}=$ $\int_{S}\left|P_{\mathrm{n}}(\boldsymbol{x})\right|^{2} d S$. Similar to the modal domain MUSIC DOA algorithm [18], we can find a set of $\boldsymbol{c}_{i}$ by calculating the autocorrelation matrix $E\left\{c c^{H}\right\}$, and then decompose $E\left\{c c^{H}\right\}$ to acquire a set of orthonormal eigenvectors and their corresponding eigenvalues. Unlike the MUSIC DOA method which utilizes the noise subspace eigenvectors, we select the signal subspace eigenvectors to be $\boldsymbol{c}_{i}$, which correspond to the significant eigenvalues $\lambda_{i}$. The eigenvalues indicate the energy distribution of the overall noise field among the basis noise patterns, and $E\left\{\left|g_{i}\right|\right\}=\lambda_{i}$.

Therefore the expected average noise power within $S$ can be calculated as

$$
E\left\{\int_{S}\left|P_{\mathrm{n}}(\boldsymbol{x})\right|^{2} d S\right\}=E\left\{\|\boldsymbol{c}\|^{2}\right\}=\sum_{i}\left\|\lambda_{i} \boldsymbol{c}_{i}\right\|^{2} .
$$

Through decomposing the noise field into basis noise patterns, we gain more insight in the dimensionality / sparsity of the noise field. A noise field of high order may have a compact representation using (10). Furthermore, additional signal analysis methods such as direction-of-arrival (DOA) estimation may be applied on the basis noise patterns to identify principal noise sources, which helps in determining optimal loudspeaker placement for ANC purposes when designing the vehicle. 


\subsection{Residual noise level estimation}

We model the characteristics of loudspeakers at each frequency bin through the wave domain channel matrix $\boldsymbol{H}$, which describes the loudspeakers' response over the entire region of interest,

$$
\boldsymbol{H}=\left[\begin{array}{cccc}
H_{00}^{1} & H_{00}^{2} & H_{00}^{3} & \ldots \\
H_{1-1}^{1} & H_{1-1}^{2} & H_{1-1}^{3} & \ldots \\
H_{10}^{1} & H_{10}^{2} & H_{10}^{3} & \ldots \\
\vdots & \vdots & \vdots & \ddots
\end{array}\right]
$$

with $H_{n m}^{j}$ being the spherical harmonic coefficient of order $n$ and degree $m$, defined in the region of interest $S$, due to the $j$ th loudspeaker playing a unit signal at one frequency. For an $N$ th order region $S$ and an array of $J$ independent loudspeakers, the size of $\boldsymbol{H}$ is $(N+1)^{2}$-by- $J$.

Since the noise field can be completely described by its eigenvectors $\boldsymbol{c}_{1}, \boldsymbol{c}_{2} .$. , we can estimate the noise cancellation performance by comparing the eigenvectors with the loudspeaker channels. In particular, we define the weighed channel matrix $\boldsymbol{T}=\boldsymbol{W} \boldsymbol{H}$, where $\boldsymbol{W}$ is the diagonal matrix defined in Section 4.1.

Then we can solve for the loudspeaker driving signal solution that minimizes (8) for each basis noise field pattern defined by $\boldsymbol{c}_{i}$, which can be derived as

$$
\boldsymbol{D}=-\left(\boldsymbol{T}^{H} \boldsymbol{T}\right)^{-1} \boldsymbol{T}^{H} \boldsymbol{c}_{i} .
$$

The residual error vector is then

$$
\boldsymbol{e}_{i}=\boldsymbol{c}_{i}+\boldsymbol{T} \boldsymbol{D}=\left(\boldsymbol{I}-\boldsymbol{T}\left(\boldsymbol{T}^{H} \boldsymbol{T}\right)^{-1} \boldsymbol{T}^{H}\right) \boldsymbol{c}_{i} .
$$

The driving signal solution (16) is essentially the Least MeanSquare Error (LMS) solution over the continuous space $S$, instead of the LMS solution based on a number of discrete spatial sampling points which is commonly used in existing car ANC systems.

We use the eigenvalues $\lambda_{i}$ as well as the original and residual noise field vectors, $\boldsymbol{c}_{i}$ and $\boldsymbol{e}_{i}$, respectively, to express the noise cancelling performance, and the overall expected noise power reduction ratio can be given using (14)

$$
e=\frac{E\left\{\int_{S}\left|P_{\mathrm{r}}(\boldsymbol{x})\right|^{2} d S\right\}}{E\left\{\int_{S}\left|P_{\mathrm{n}}(\boldsymbol{x})\right|^{2} d S\right\}}=\frac{\sum_{i}\left\|\lambda_{i} \boldsymbol{e}_{i}\right\|^{2}}{\sum_{i}\left\|\lambda_{i} \boldsymbol{c}_{i}\right\|^{2}},
$$

where the term $\boldsymbol{c}_{i}$ in (18) can be omitted since $\boldsymbol{c}_{i}$ are orthonormal.

\section{EXPERIMENTAL RESULTS}

\subsection{Experiment setup}

In this experiment, we use the method developed in the previous sections to analyze the potential noise cancellation performance of the loudspeakers installed in a car (2005 Ford Falcon XR6 sedan).

We use an Eigenmike to measure the in-car noise field; the region of interest is chosen to be a spherical area with $10 \mathrm{~cm}$ radius, located at the head position of the frontal passenger seat. The radius of the region is larger than that of the EigenMike $(4.2 \mathrm{~cm})$, therefore we only analyze the sound field for frequencies below 500 $\mathrm{Hz}$, within this frequency range, only the 0 th and 1 st order sound field harmonics are active inside the region of interest [19], which can be reliably measured by the Eigenmike placed in the center of the region. Also, spectral analysis of the in-car noise indicate that the majority of the noise power lie below $500 \mathrm{~Hz}$ (an example of

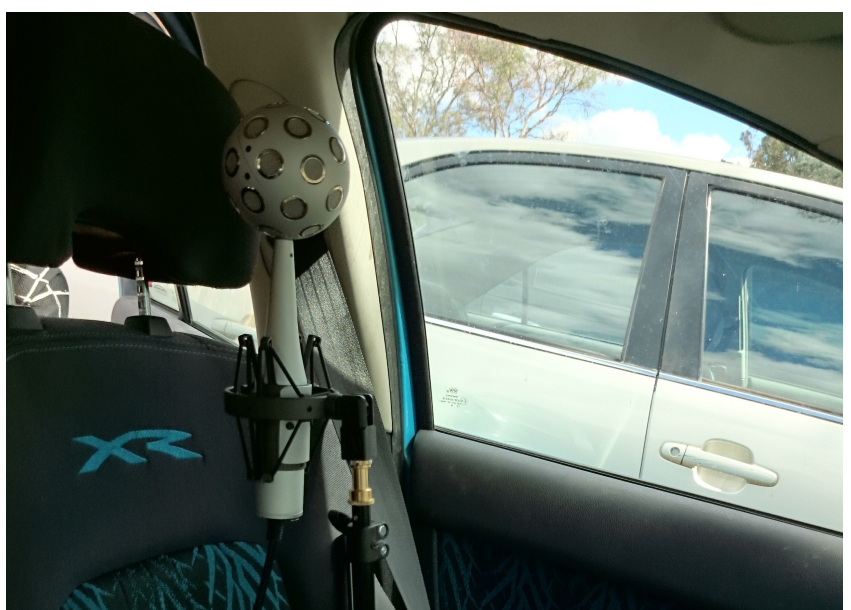

Figure 1: Picture of the EigenMike installed in a Ford Falcon XR6.

the noise spectrum is shown in FIG 3), thus the noise cancelling performance within this frequency band is indicative of the overall cancelling quality.

The vehicle has four full-band loudspeakers installed, two of which are integrated in either of the front doors, while the other two are placed behind each rear seat. Unfortunately, the car's audio system can only play stereo signals, which means the two loudspeakers on either side cannot be driven separately, and always play the same signal.

In order to characterize the noise field, we record the in-car noise under various driving conditions. We also recorded the noise fields due to engine and air-conditioner while the car is stationary. For each driving condition, a 10-second-long recording is separated into 100 snapshots, we then calculate the sound field coefficients for each snapshot and at every frequency bin, and finally calculate the coefficient covariance matrix of all the 100 snapshots. The covariance matrix used as the estimation of $c c^{H}$, and is used for further data analysis.

The loudspeaker channel matrix is obtained by measuring the spatial response at the region of interest due to the left channel and right channel separately using the Eigenmike, the sound field coefficients for each frequency bin are calculated in the same way as the noise field samples. The 1st order sound field and the stereo speaker system result in a 4-by-2 channel matrix for each frequency bin.

When calculating the residual noise field vector $\boldsymbol{e}_{i}$, we include a small regularization parameter $\beta$, such that (17) becomes

$$
\boldsymbol{e}_{i}=\left(\boldsymbol{I}-\boldsymbol{T}\left(\boldsymbol{T}^{H} \boldsymbol{T}+\beta \boldsymbol{I}\right)^{-1} \boldsymbol{T}^{H}\right) \boldsymbol{c}_{i},
$$

with $\beta=0.01$. The regularization prevents severe ill-conditioning of the matrix inversion, thereby preventing the occurrence of very high secondary loudspeaker volumes.

\subsection{Experimental data analysis}

By diagonalizing the estimated coefficient covariance matrices acquired from the recordings from various driving conditions, we obtained the eigenvalues for every case and each frequency bin. The eigenvalues are given in Table 1 for the freeway driving condition and a pure engine noise recording. For the freeway recording, the car was driven on a freeway at $100 \mathrm{~km} / \mathrm{h}$, with air conditioning 


\begin{tabular}{|c|c|c|c|c|c|}
\hline $100 \mathrm{~km} / \mathrm{h}$ & $100 \mathrm{~Hz}$ & $200 \mathrm{~Hz}$ & $300 \mathrm{~Hz}$ & $400 \mathrm{~Hz}$ & $500 \mathrm{~Hz}$ \\
\hline$\lambda_{1}$ & 137.7 & 29.58 & 18.43 & 15.02 & 9.360 \\
\hline$\lambda_{2}$ & 6.578 & 1.049 & 1.286 & 1.270 & 1.134 \\
\hline$\lambda_{3}$ & 2.610 & 0.651 & 0.814 & 0.800 & 0.679 \\
\hline$\lambda_{4}$ & 1.475 & 0.418 & 0.645 & 0.596 & 0.414 \\
\hline \hline Engine Only & $100 \mathrm{~Hz}$ & $200 \mathrm{~Hz}$ & $300 \mathrm{~Hz}$ & $400 \mathrm{~Hz}$ & $500 \mathrm{~Hz}$ \\
\hline$\lambda_{1}$ & 74.50 & 50.97 & 17.65 & 8.697 & 5.029 \\
\hline$\lambda_{2}$ & 2.217 & 1.807 & 0.930 & 0.997 & 0.578 \\
\hline$\lambda_{3}$ & 0.862 & 0.706 & 0.496 & 0.574 & 0.442 \\
\hline$\lambda_{4}$ & 0.557 & 0.400 & 0.341 & 0.369 & 0.172 \\
\hline \multicolumn{5}{|l}{}
\end{tabular}

Table 1: Table of noise field eigenvalues for freeway driving condition and pure engine noise

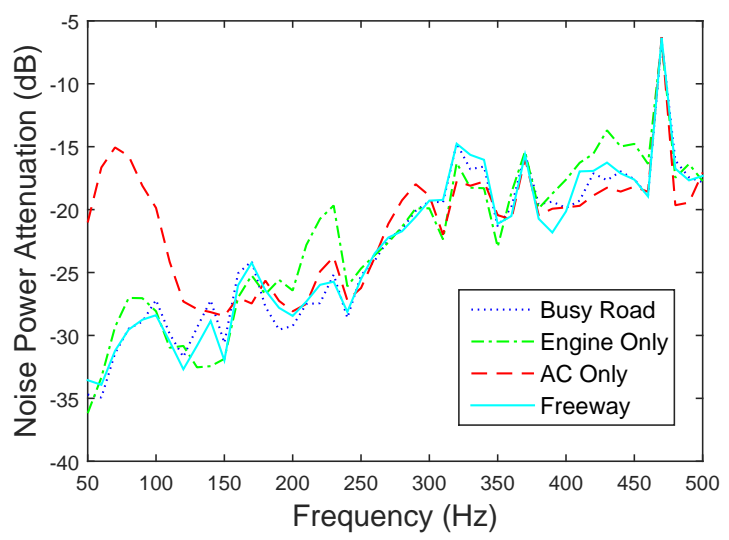

Figure 2: Noise power spectrum attenuation for 4 different driving conditions.

turned to low; for the engine noise recording, the car was parked in a quiet place with air conditioning switched off, and the engine ran at various rpm during the recording.

From Table 1 we see that the eigenvalues decay quickly as the frequency increases, which indicates the shape of the noise spectrum, where the lower frequencies are more dominant.

Furthermore, one may notice that the first eigenvalues for both cases and each frequency bin are much larger than the other 3 eigenvalues, this is particularly noticeable at lower frequencies, and the same phenomenon is observed in all the other driving scenarios. In Section 4.1 we showed that each eigenvector of the covariance matrix corresponds to a specific spatial sound field pattern, with the relative importance of each pattern indicated by its eigenvalue. This result shows that there is one dominant noise pattern in the region of interest for each frequency bin. Therefore we expect that the lower frequency noise fields can be seen as sparse, thus controlling such sound fields may require only a small number of well-placed loudspeakers which can nicely reproduce the dominant noise pattern.

FIG. 2 plots the noise power attenuation for four different driving conditions, with the values calculated using (18). In addition to the freeway recording and the engine noise recording, the "Busy Road" recording was taken while driving on a 3-lane road at moderate speed with multiple vehicles passing by; while the "AC only" recording was taken with the car parked in a quiet place and engine idle, the air conditioning turned to maximum.

FIG. 2 indicates that for most cases, the noise cancelling performance is relatively consistent, with the attenuation reducing gradually from $30-35 \mathrm{~dB}$ at $50 \mathrm{~Hz}$ to $15-20 \mathrm{~dB}$ at $500 \mathrm{~Hz}$. This

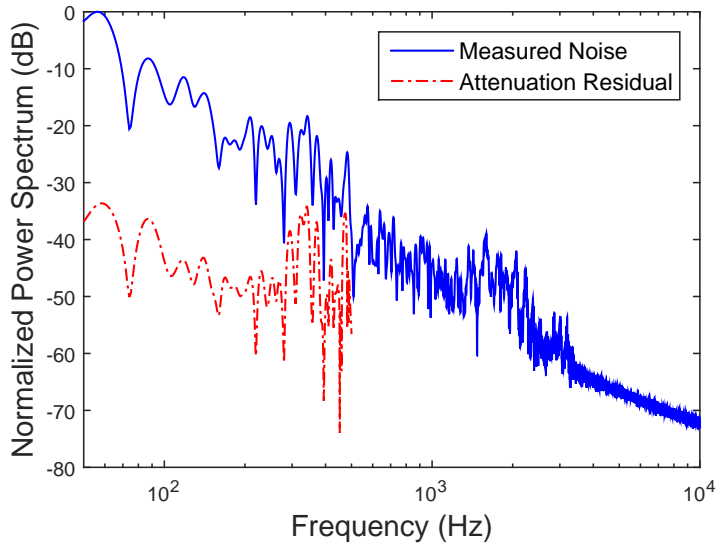

Figure 3: Comparison of average noise field power spectrum before and after cancellation.

frequency-dependent performance is expected since the noise field is expected to be more complicated and harder to reproduce/cancel when the wavelength is shorter. We also notice that the noise field due to air conditioning is particularly difficult to cancel at $50-100$ $\mathrm{Hz}$, compared to other scenarios. We expect this is because the noise field due to AC is less similar to that of the loudspeakers, compared to other noise sources. One may also notice the common peak in all cases at $470 \mathrm{~Hz}$, clearly at this frequency, the loudspeakers are unable to reproduce the noise fields very well.

We also include FIG. 3 which depicts the overall noise spectrum without attenuation, and the expected residual noise spectrum if the in-car loudspeakers are employed to cancel the noise field. The original noise spectrum is recorded while driving at $70 \mathrm{~km} / \mathrm{h}$ with air conditioning at minimum. The attenuation is cut off at $500 \mathrm{~Hz}$. We can see from the figure that the most dominant noise frequencies can be effectively cancelled by the integrated loudspeakers, resulting in a much quieter sound field within the region of interest.

In general, we can conclude that the integrated loudspeakers are capable of cancelling the noise field within our defined region of interest at the front passenger seat. However, we would expect the performance to degrade should the noise cancellation be carried out for multiple seats. Nevertheless, a proper in-car ANC system would be able to drive the four loudspeakers separately, which provides extra degrees of freedom for the loudspeaker channels, thereby promoting the overall performance of the system.

\section{CONCLUSION}

This paper presents an expression to represent the average noise power within a spherical region, as well as a method to characterize the spatial noise field within the region. Using the channel information of the secondary loudspeakers, we derive the expected spatial noise cancelling performance of the integrated loudspeakers in a car.

Through analysis of the in-car noise data acquired from field tests using the method we proposed, we show that the noise field at low frequencies are relatively sparse, and that the car's integrated loudspeakers are capable of cancelling the in-car noise field below $500 \mathrm{~Hz}$ around a typical head position. 


\section{REFERENCES}

[1] P. Nelson and S. Elliott, Active control of sound. Academic press, 1991.

[2] S. Hasegawa, T. Tabata, A. Kinoshita, and H. Hyodo, "The development of an active noise control system for automobiles," SAE Technical Paper, Tech. Rep., 1992.

[3] S. Kuo and D. Morgan, "Active noise control: a tutorial review," Proceedings of the IEEE, vol. 87, no. 6, pp. 943-973, 1999.

[4] C. Bohn, A. Cortabarria, V. Härtel, and K. Kowalczyk, "Active control of engine-induced vibrations in automotive vehicles using disturbance observer gain scheduling," Control Engineering Practice, vol. 12, no. 8, pp. 1029-1039, 2004.

[5] S. J. Elliott and P. A. Nelson, "The active control of sound," Electronics \& communication engineering journal, vol. 2, no. 4, pp. 127-136, 1990.

[6] H. Sano, T. Inoue, A. Takahashi, K. Terai, and Y. Nakamura, "Active control system for low-frequency road noise combined with an audio system," IEEE Transactions on Speech and Audio Processing, vol. 9, no. 7, pp. 755-763, 2001.

[7] S. J. Elliott and T. J. Sutton, "Performance of feedforward and feedback systems for active control," IEEE Transactions on Speech and Audio Processing, vol. 4, no. 3, pp. 214-223, 1996.

[8] J. Cheer and S. Elliott, "Multichannel control systems for the attenuation of interior road noise in vehicles," Mechanical Systems and Signal Processing, vol. 60, pp. 753-769, 2015.

[9] S. Oh, H. Kim, and Y. Park, "Active control of road booming noise in automotive interiors," The Journal of the Acoustical Society of America, vol. 111, no. 1, pp. 180-188, 2002.

[10] J. I. Mohammad, S. J. Elliott, and A. Mackay, "The performance of active control of random noise in cars," The Journal of the Acoustical Society of America, vol. 123, no. 4, pp. 1838-1841, 2008.
[11] J. Cheer and S. J. Elliott, "The design and performance of feedback controllers for the attenuation of road noise in vehicles," International Journal of Acoustics and Vibration, 2014.

[12] Y. Kajikawa, W.-S. Gan, and S. M. Kuo, "Recent advances on active noise control: open issues and innovative applications," APSIPA Transactions on Signal and Information Processing, vol. 1, 2012.

[13] A. Barkefors, S. Berthilsson, and M. Sternad, "Extending the area silenced by active noise control using multiple loudspeakers," in IEEE International Conference on Acoustics, Speech and Signal Processing (ICASSP). IEEE, 2012, pp. 325-328.

[14] S. Spors and H. Buchner, "An approach to massive multichannel broadband feedforward active noise control using wavedomain adaptive filtering," in IEEE Workshop on Applications of Signal Processing to Audio and Acoustics, Oct 2007, pp. 171-174.

[15] E. G. Williams, Fourier Acoustics: Sound Radiation and Near field Acoustical Holography. USA: Academic, 1999.

[16] D. B. Ward and T. Abhayapala, "Reproduction of a planewave sound field using an array of loudspeakers," IEEE Transactions on Speech and Audio Processing, vol. 9, no. 6, pp. 697-707, Sep 2001.

[17] S. Elliott, "A review of active noise and vibration control in road vehicles," ISVR Technical Memorandum, Technical Report 981, 2008.

[18] X. Li, S. Yan, X. Ma, and C. Hou, "Spherical harmonics MUSIC versus conventional MUSIC," Applied Acoustics, vol. 72, no. 9 , pp. $646-652,2011$.

[19] R. A. Kennedy, P. Sadeghi, T. D. Abhayapala, and H. M. Jones, "Intrinsic limits of dimensionality and richness in random multipath fields," IEEE Transactions on Signal Processing, vol. 55, no. 6, pp. 2542-2556, 2007. 